\title{
Correlation Between the Gut Microbiome and Immunotherapy Response in Inflammatory Bowel Disease: A Systematic Review of the Literature
}

\author{
Katarzyna Karpinska-Leydier ${ }^{1}$, Jashvini Amirthalingam ${ }^{2}$, Khadija Alshowaikh ${ }^{3}$, Anuruddhika Iroshani
} Jayarathna $^{2}$, Divya Bala Anthony Manisha R. Salibindla ${ }^{4}$, Gokul Paidi ${ }^{1}$, Huseyin Ekin Ergin ${ }^{5}$

1. Internal Medicine, California Institute of Behavioral Neurosciences \& Psychology, Fairfield, USA 2. Neurology, California Institute of Behavioral Neurosciences \& Psychology, Fairfield, USA 3. Obstetrics and Gynecology, California Institute of Behavioral Neurosciences \& Psychology, Fairfield, USA 4. Pathology, California Institute of Behavioral Neurosciences \& Psychology, Fairfield, USA 5. General Practice, California Institute of Behavioral Neurosciences \& Psychology, Fairfield, USA

Corresponding author: Katarzyna Karpinska-Leydier, kkarpinskaleydier@gmail.com

\begin{abstract}
Inflammatory bowel disease (IBD) is an autoimmune disease associated with dysbiosis within the gastrointestinal tract. Characteristic taxonomic shifts of microbial populations are observed in disease progression and remission; however, despite similarities, there are many differences among individuals presenting with IBD including IBD subset, clinical course, and response to therapy. Much is still unknown about how these taxonomic shifts interact with immunotherapy and how genetic variants contribute. In this systematic review, we aimed to compile information on the interactions of the gut microbiome with immunotherapy in the course of disease and treatment of IBD patients. This systematic review was conducted as per the Preferred Reporting Items for Systematic Reviews and Meta-Analyses (PRISMA) guidelines and the PubMed database was methodically screened for literature search including keywords and Medical Subject Headings (MeSH) terms for relevant articles.
\end{abstract}

The quality appraisal was completed using the Cochrane Tool, Newcastle-Ottawa checklist, and the Scale for the Assessment of Narrative Review Articles (SANRA) checklist, as appropriate, and 11 relevant articles were included in this systematic review. Our review concludes that although there are characteristic taxonomic shifts between diseased and healthy patients, genetic variants are an important consideration in the predictive quality of disease and treatment decisions. The comparison between interactions of microbial populations and treatment in addition to the role of genetic variants may provide insight into treatment non-responders. Due to our limitations in current knowledge including the complexity of the microcosm, ethnic genetic variations among human populations, and our focus on relevant articles published in English over the past six years, we may have missed relevant studies. Future studies should focus on the comparison between Western and other cultural populations as well as further implementation of Genome-Wide Association Studies (GWAS) in clinical predictability.

Received 06/08/2021

Review began 07/03/2021 Review ended 07/12/2021 Published 08/01/2021

๑) Copyright 2021

Karpinska-Leydier et al. This is an open access article distributed under the terms of the Creative Commons Attribution License CC-BY 4.0., which permits unrestricted use, distribution, and reproduction in any medium, provided the original author and source are credited.

Categories: Internal Medicine, Gastroenterology

Keywords: inflammatory bowel disease, crohn's disease (cd), gastrointestinal microbiome, intestinal microbiota, gut flora, immune modulation therapy, ulcerative colitis (uc)

\section{Introduction And Background}

One of the major functional components of the gastrointestinal system is the delicately balanced microbiota within it. Dysbiosis, the unfavorable alteration of this commensal, is linked to several human pathologies $[1,2]$. This imbalance has been implicated in chronic inflammation, including autoimmune disease in the form of inflammatory bowel disease (IBD) [3]. The two subsets of IBD are different in that ulcerative colitis (UC) continuously affects the large intestine and rectum with epithelial inflammation and superficial ulcers, while Crohn's disease (CD) unevenly affects both the large and small intestines with fullthickness ulceration $[3,4]$. The understanding of the microbiome and its role in human health largely still lacks elucidation, however, the use of innovative technologies is improving our understanding [5]. Genomewide association studies (GWAS) have identified novel genetic variants associated with differing traits such as early-onset CD (CTLA-4) [6,7].

The pathogenesis of IBD is multifactorial involving genetics, microbiota, and environment interacting with the host immune system $[1,8,9]$. Reflective of this complexity is the dynamic change with which different treatments alter both the host immune response and the gut microcosm $[1,10,11]$. For this reason, several treatment modalities exist, however, the taxonomic shift relative to treatment is still largely uncategorized [12]. Despite the differences between IBD subsets, both result from autoimmune tissue destruction, therefore, targeted therapy includes immunomodulators and immunosuppressants [3]. Tumor necrosis factor (TNF)-targeted treatment is effective but a large percentage of patients are incomplete or non-responders [13]. Just as the interaction between the gut microbiota and the host immune system is a 
factor in disease development, the changes in microbiomes throughout this progression may be related to the inadequate response [13].

Reduced inflammation in anti-TNF-treated patients results correspondingly from modulation of the gut microbiome which reverts to a composition comparable to that of healthy individuals including reduced Enterobacteriaceae coli (E. coli) and Ruminococcus species, as well as increased proportions of Bacteroidetes and Firmicutes [13]. Similarly, combinations of antibiotics have been clinically shown to improve flares in both adult and pediatric IBD patients likely by decreasing mucosal inflammation via the alteration of intestinal microbiota [14]. However, no single bacterial species have been implicated in disease causation, furthering that a non-specific imbalance coupled with other stressors lead to disease processes, including the less explored virome and fungal gut components [15-17].

Our capacity to evaluate the microbiome as a functional (or dysfunctional) component of the gastrointestinal system has impacted the way we can treat IBD including both antibiotics and probiotics, the latter intended to confer a beneficial population of bacteria often through dietary contribution for the relief of functional gut disorders [18-20]. A mainstay of IBD treatment is focused on immunosuppression such as in UC with the implementation of mesalamine, glucocorticoids, thiopurines, and biologic agents [21,22]. Despite this, immunosuppressive therapies to date are not known to sustain remission for UC patients [21].

The gut microbiome is seemingly involved in more than macromolecular digestion, as the pathogenic role of dysbiotic inflammation has been linked to other comorbidities such as mental illness, including depression $[23,24]$. Inflammatory cytokines and acute-phase proteins such as interleukin (IL)-6 and Creactive protein (CRP) were evaluated through meta-analysis showing an increased incidence of depressive symptoms [23]. Co-morbid depressive symptoms predict poor disease course in IBD and are inconsistently improved by psychological treatments, inferring that disease progression including co-morbid depression may stem from the effects of treatment on gut flora [23,25]. Immunomodulators have shown meaningful changes in microflora populations with clinical improvement but more needs to be done to understand the impact of immunotherapy treatments on the gut microbiome to explain why these treatments may work in some patients but remain ineffective or partially effective in other patients. This systematic review explores the current literature on the association between immunotherapy and the microbiome of IBD patients.

\section{Review}

\section{Methods}

Protocol

This systematic review was performed and reported in agreement with the Preferred Reporting Items for Systematic Reviews and Meta-Analyses (PRISMA) [26].

\section{Inclusion/Exclusion Criteria}

This literature search was performed to identify studies that defined immunotherapy and the gut microbiome in all-aged patients with IBD. The criteria used to search for eligible studies included the following: (1) response to immunotherapy in IBD, (2) variations in the gut microbiome in IBD, (3) variations in the gut microbiome in immunotherapy, and (4) variations in immunotherapy response in patients where the gut microbiome was studied. The studies that reported other illnesses or immunotherapy used in other illnesses were excluded as they were outside the scope of the extant study. The study types included were limited to randomized control trials (RCT), observational/non-randomized studies, and review articles either systematic in nature or unspecified.

\section{Search Strategy}

A methodical search of the PubMed [27], PubMed Central (PMC), and MEDLINE databases was conducted on April 29, 2021. The search for relevant studies using generic keywords (Immunotherapy) AND ((Gastrointestinal Microbiome) OR (Microbiota)) AND ((Inflammatory Bowel Disease) OR (Crohn Disease) OR (Ulcerative Colitis)) was done and 108 studies were identified. The relevant Medical Subject Headings (MeSH) terms and keywords "inflammatory bowel disease," "complications" "diet therapy" "drug therapy" "genetics" "immunology" "metabolism" "microbiology" "therapy" were used in various combinations using Boolean operators like "AND" and "OR," and 5,860 studies were identified. The inclusion/exclusion criteria were applied through an automation tool and records from January 2015 to April 2021 were identified. The search extended to studies published in the English language for human participants and returned 1,878 relevant studies. In accordance with the inclusion/exclusion criteria, gray literature was not included in this study. The results of the search strategy with respective keywords are displayed in Table 1 and Table 2. 


\section{Cureus}

Keywords

(Immunotherapy) AND ((Gastrointestinal Microbiome) OR (Microbiota)) AND

((Inflammatory Bowel Disease) OR (Crohn Disease) OR (Ulcerative Colitis))
Total Articles after Inclusion/Exclusion

Articles Criteria Applied by Automation

$108 \quad 32$

TABLE 1: PubMed Database search results with regular keywords.

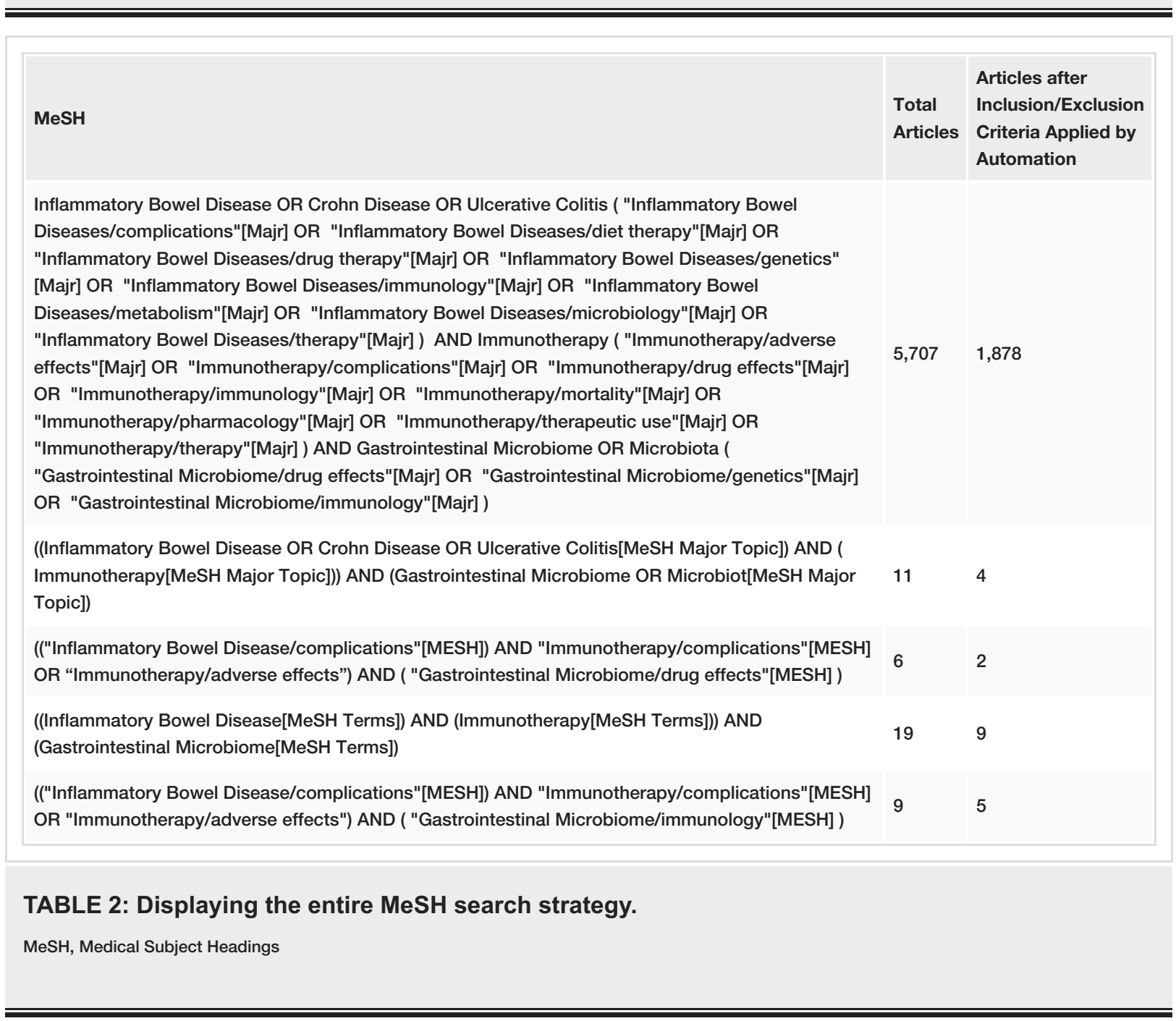

Data Extraction

Articles were screened by full-text, abstracts, and titles independently by two authors, KK and JA. Both parties reviewed and scrutinized the relevance, eligibility, and quality of the studies independently. Where necessary, differences in judgment were addressed through mutual discussion.

Bias Evaluation and Data Explication

The quality appraisal was conducted to only include moderate-to-high quality studies in the final investigation and was conducted with the following tools: Cochrane Tool (randomized control trial), Newcastle-Ottawa checklist (observational/non-randomized control trial), and the Scale for the Assessment of Narrative Review Articles (SANRA) checklist (traditional review articles).

\section{Results}

Search Outcome

A total of 5,860 papers were identified through a field search of PubMed, PMC, and MEDLINE. After the 


\section{Cureus}

application of the inclusion/exclusion through the automation tool, 1,930 articles remained for further processing; 27 duplicates were removed using EndNote Basic and 1,903 articles were screened through title and abstract for relevance. 1,753 articles were excluded and the resulting 152 relevant articles were screened in-depth with the inclusion/exclusion criteria in the current analysis. A total of 12 relevant studies were assessed for quality appraisal and 11 moderate-to-high quality studies were included in this systematic review for discussion. Figure 1 demonstrates the PRISMA flow diagram in conducting the search for this review.

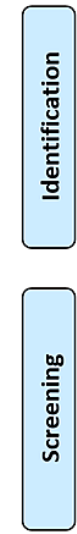

Records identified through PubMed, PMC, and MEDLINE with

keywords and $\mathrm{MeSH}$ ( $n=5,860$ )

Additional records identified through other sources $(n=0)$
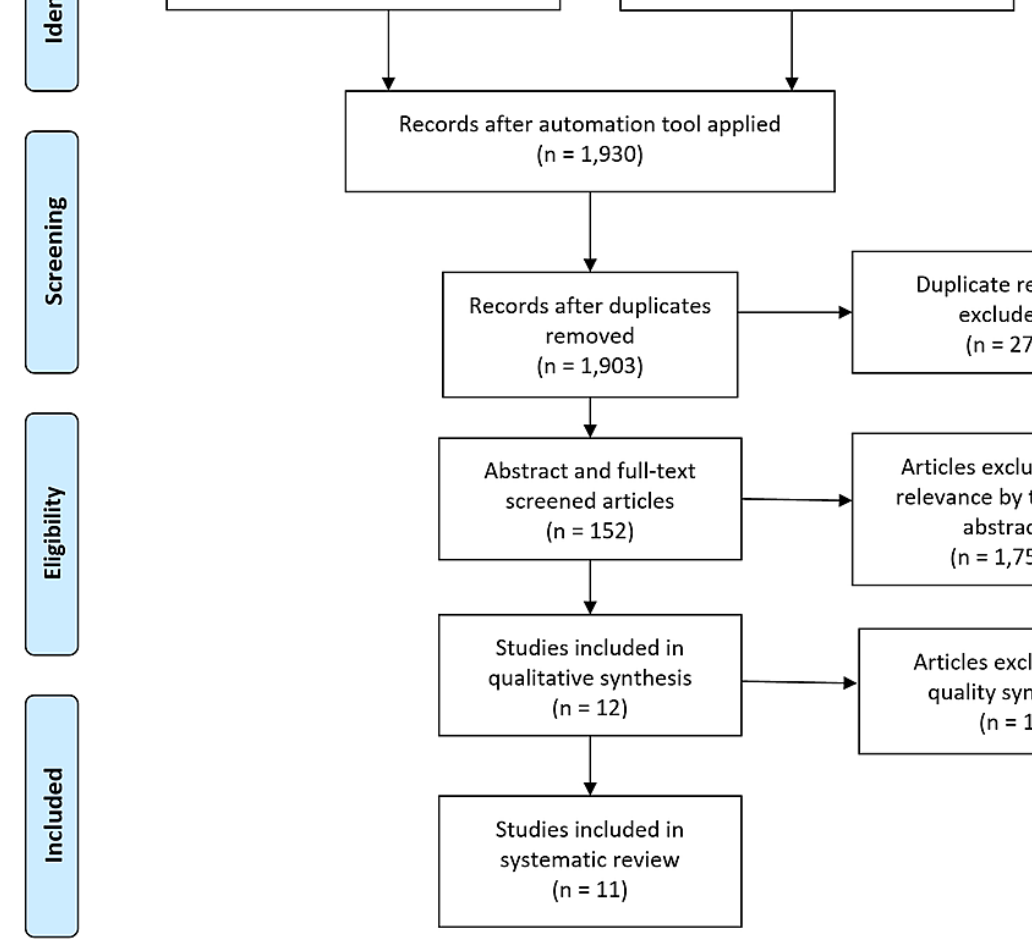


\section{Cureus}

O'Reilly et 2020 Randomized Assess the effect of using colon-targeted delivery al. [29] control trial system CyA formulation on the composition and functionality of the gut microbiota.

Report an integrative analysis on CD-related

Li et al. [30] 2019 Observational genetic defects in innate immune function and the study

two consecutive patient cohorts.

Characterize the microbiota of patients with CC

Carstens et al. [31] 201 Observational study patients.
Schirmer et al. [32] 018

Observational study

Evaluate the role of the gut microbiome in disease course for new-onset, treatment-naïve, pediatric UC patients, as part of the PROTECT Study.

Grigg and

Sonnenberg 2017 Review article [33]

Guariso and

Gasparett 2017 Review article [34]

Hodzic et al. [3]

2017 Review article

Literature review on the role of IL-33 in the regulation of intestinal immunity, involvement in the intestinal disease, and implication in potential therapeutics.

Investigate the impact of high fat and high sugar diets on mice to better understand the mechanisms involved in modulation of host

Agus et 2016 Randomized physiology within gut inflammation and microbiota al [35]. 2016 control trial composition and function compared to human IBD patients. Histological disease activity was measured using human CD mucosal biopsy specimens.

Analyze the variation of intestinal microbial

Eun et al. [36] stud community structure in KCD patients by comparing fecal and mucosal tissue samples using metagenomic analysis with healthy controls. microbiota composition; the ex vivo colon model demonstrated relevance in predicting in vivo impact of drugs on the microbiota.

\section{Confirm significant effect of IBD}

phenotype, C. difficile infection, and NOD2 genotype on ileum-associated microbiota; additional IBD-related genotypes are associated with changes in ileal microbial composition.

Common mechanisms may underlie the pathogenesis of CC and IBD, as shifts in key taxa were similar; a specific fecal microbiome is seen in CC patients with active disease and corticosteroid treatment, while the microbiome of CC patients in remission resembled healthy controls.

Baseline and longitudinal microbial trends involved in the progression of disease and remission were observed; there is evidence that the microbiome impacts treatment efficacy in UC and microbial biomarkers could inform treatment strategies.

Gut microbiota impacts the quality of immune function and correspondingly, the immune system informs the geography and demographics of microbiota; therapies that modulate or re-establish beneficial interactions are promising treatments for inflammatory disease.

Many children with IBD do not respond to current treatment options which target known pathways of an intricate disease process; the development of reliable prognostic biomarkers is an essential leap towards tailored treatment.

The role of IL-33 is context-dependent: its behavior in steady-state is different than in epithelial injury. IL-33 has a role in signaling within IBD that is still being understood and there is further need to recognize it within the context of potential immunomodulatory therapeutics.

The high fat and high sugar diet created an inflammatory environment in the gut correlating with dysbiosis, however, the expression of GPR43 (SCFA receptor) was reduced in mice with this diet and is similarly reduced in CD patients compared with healthy controls.

Intestinal microbial community structure in KCD patients was shown to be similar to that of Western CD patients as demonstrated by $16 \mathrm{~S}$ rRNA sequencing anti-TNF treatment may affect microbial community structure.

Dysbiosis in CD also includes aberrations 


\section{Cureus}

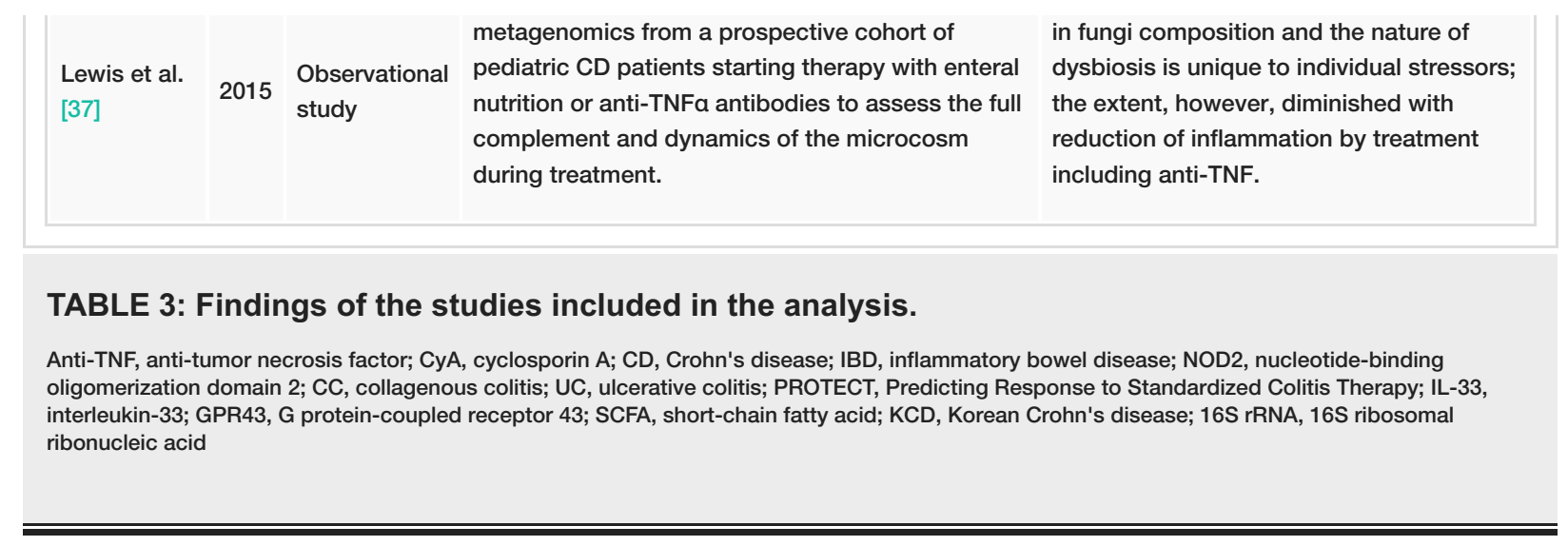

\section{Discussion}

We studied 11 previously published articles to understand the relationship between human gastrointestinal tract microbial populations and the use of immunotherapy in IBD-afflicted patients. In this review, we aimed to gain an updated understanding of this relationship and how disease recurrence or remission is achieved inconsistently among patients.

\section{Microbiome Aberrations in Diseased and Healthy States}

The pathogenesis of IBD is multifactorial but the gut microbiome is a significant component of chronic inflammatory disorders as shown by the mechanistic understanding of current treatments and documented population shifts. A study conducted by Carstens et al. compared fecal samples between patients with collagenous colitis (CC) and patients with CD and UC in addition to healthy controls [31]. Their findings demonstrated lower microbial diversity among CC patients as compared to healthy controls which resembled the findings of IBD patients, including a shift in the Ruminococcaceae family (a member of the Firmicutes phylum) [31]. Interestingly, a study conducted by Eun et al. observed that Firmicutes populations declined in IBD-afflicted patients in both Korean and Western subjects despite their differences in dietary habits (including proinflammatory high fat and confectionary diets typical in Western cuisine) which are known to affect intestinal microorganisms [35,36]. Beneficial host-microbiota interactions rely on multiple factors including diet and systemic immunoglobulin responses [33]. The role of Firmicutes, in particular, is implicated in gut health as an energy source and inflammatory modulation via fermentation of dietary fiber to produce important short-chain fatty acids (SCFA) such as acetate, propionate, and butyrate [36]. Patients with IBD are exposed to both dietary changes and antibiotics which invariably alter microbial community structures, although the precise effects are not completely understood [37]. Despite relative similarities between microbial demographics in CC as compared with IBD, they are phenotypically different diseases. Furthermore, while dysbiosis commonly refers to bacterial microbial structures, other domains within the gut microbiome are less studied and may contribute to unaccounted variation between patients [37].

Similarly, Eun et al. compared intestinal microbial communities in Korean CD (KCD) patients with that of Western CD patients and healthy controls [36]. Their deep sequencing study demonstrated dysbiosis patterns in the KCD patients that were comparable to their Western counterparts, including decreased microbial diversity and proportionately increased Proteobacteria and Fusobacteria populations while Firmicutes and Bacteroides populations declined [36]. Importantly, the microbial demographics in this study differed from the healthy controls where both mucosal tissue and fecal samples showed Escherichia and Shigella species abundantly in the diseased patients which coincide with known literature involving the role of E. coli strains in inducing inflammation $[35,36]$.

A study by Lewis et al. used shotgun metagenomic sequencing to analyze fecal samples from a prospective cohort of CD-afflicted pediatric patients beginning either enteral nutrition or anti-TNF $\alpha$ antibodies and observed that dysbiosis occurred through not only bacterial composition but also differences in gene representation, higher fungal expanse, and increased volume of human DNA in the stool [37]. They further discuss that the independent effects of antibiotics, diet, and inflammation are reflective of mechanistic differences and, although dysbiosis in $\mathrm{CD}$ is common, the quality of these aberrations is unique to the stressor [37]. However, there has been conflicting evidence surrounding UC and microbial diversity which has sometimes been indifferent compared with healthy individuals [31].

The relative success of fecal microbial transplant (FMT) is considered low or inconclusive at present, with minimal data to support recommendations for IBD treatment, however, ongoing medical management is aimed at modulating inflammation and altering microbiome expression [34]. Multiple studies have demonstrated that the diminished diversity of taxa in inflammatory disease states often have similar profiles. While it might be expected that similar microbial profiles would produce a similar disease phenotype, perhaps finding differences in the overall microcosm profiles (including viruses and fungi) between disease states or other host differences such as genetic polymorphisms may explain differing 
disease phenotypes under similar aberrant microbial profiles.

Gene Expression Implicated in Disease

Gene expression in both the host and the gut microcosm interact with environmental stressors to create a favorable (or unfavorable) condition for the development of inflammatory autoimmune diseases. A study by Sakurai et al. evaluated the association between long-term remission in UC after discontinuation of antiTNF therapy, mucosal microbiota, and gene expression profiles [28]. They used machine learning with IBD GWAS data as a predictive tool in a similar method as is used to predict cancer susceptibility, recurrence, and survivability [28]. UC follows a relapsing and remitting course where machine learning, as a predictive tool, could advise patients on therapy discontinuation [28]. They found that gene expression in the nonrelapse UC group had substantially higher gene expression of ALIX, also known as programmed cell death 6interacting protein (PDCD6IP), and SLC9A3 (Solute carrier family 9 member A3) compared with the relapse group [28]. Furthermore, it is speculated that expression of ALIX, IL22RA (interleukin-22 receptor subunit alpha-1), LGR6 (leucine-rich repeat-containing G protein-coupled receptor 6), and SLC9A3 may prevent UC flares since these genes were downregulated after anti-TNF therapy in the relapse group while in heat shock proteins and ITGA7 (integrin alpha 7) were upregulated in this group [28].

Another study by Li et al. explored abnormal host-microbial interactions through genetic susceptibility, focusing on CD-related genetic defects in innate immune function and specifically the ileal microbiome by comparing phenotypic variants of CD comprised of ileum-affected, colitis, or non-IBD patients [30]. This group's previous work examined nucleotide-binding oligomerization domain-containing protein 2 (NOD2) and protein-coding gene, autophagy-related 16-like 1 (ATG16L1), polymorphisms on ileal microbial populations including the link between NOD2 genotypes and C. difficile infection [30]. Further, differential gene expression altering Paneth cell function was found to promote dysbiosis [30]. In the current study, higher representation of the Proteobacteria phylum was linked to the NOD2R genotype independent of IBD phenotype even in disease unaffected ileal mucosa and may be related to the observation of relatively lower Proteobacteria abundance in unaffected mucosa compared with adjacent disease affected mucosa in ileal CD phenotypes [30]. The differences between these mucosal neighborhoods strengthen the concept of dysbiosis in normal-appearing mucosa [30].

Genetic susceptibility may vary among different ethnic populations. Susceptibility loci differ between Asian and Western populations and while NOD2 and ATG16L1 do not show replication results in Asian populations, the incidence of CD in Asian populations is increasing [36]. ATG16L2 mutations have been observed in KCD patients and are known to regulate bacterial signaling, which (although different from the Western mutations), is similarly involved in bacterial modulation which reinforces the concept that abnormal interactions between gut microflora and susceptible host genes promote CD development [36]. IBD phenotype and genetic variation underly, in part, how the host immune system interacts with local flora; furthermore, although certain identified genes can help predict remission, a methodology for how these factors interact with therapy needs further elucidation. Machine learning provides a hopeful framework for a predictive approach to disease course but further work on the mapping of the gut microbiome, genetic variability, and their interaction with therapy is needed to provide solutions for disease recurrence and treatment non-responders.

Interactions between the Microbiome and Immunotherapy

The use of both probiotics and antibiotics are broadly used to treat gastrointestinal disorders by the targeted alteration of microbial populations, however, microbial communities at the intestinal level have been shown to change throughout immunomodulation in IBD by either direct or indirect causes. Intestinal microbiota can contribute to xenobiotic metabolism which may influence drug bioavailability and ultimately the efficacy and safety of oral medications [29]. A study by O'Reilly et al. evaluated a colon-targeted delivery system (encapsulated SmPill minispheres) for cyclosporine A (CyA) on the composition and functionality of gut microbiota [29]. Steroid-refractory UC can be treated with systemic CyA and infliximab, however, CyA has a narrow therapeutic window and requires monitoring [29]. In this ex vivo colon model (and the conducted in vivo pilot of healthy volunteers), gut microbiota composition was not affected but the impact of immunosuppression on the host as a downstream influence on microbiota could not be ruled out [29] Furthermore, SmPill formulations increased the concentration of n-butyrate, which is known to positively correlate with host health [29].

A lack of immunosuppressant maintenance treatment and poor mucosal healing is associated with an increased risk of relapsing in UC patients who discontinue anti-TNF therapy [28]. The maintenance of gut homeostasis is, in part, maintained by Dore sp. and Lachnospira sp. genera, which produce beneficial SCFAs [28]. The Sakurai et al. study reported that gut microbial surveillance at week 0 and 24 post-anti-TNF therapy cessation are predictive of sustained remission after initial anti-TNF therapy remission [28]. Conversely, pro-inflammatory Fusobacterium sp. and Veillonella dispar are associated with relapse [28].

Immune education, development, and response are impacted by the microbiome and its products. As part of the PROTECT study, Schirmer et al. evaluated taxonomic shifts on treatment efficacy of treatment naïve, 
pediatric UC patients [32]. They found that patients with severe disease, compared with their baseline, had higher populations of oral cavity bacteria than expected which infers that severe disease may correlate with a propensity of aberrant colonization compared with resistant, healthy guts [32]. Expression of virulence genes by microbes may also aid in abnormal colonization and exacerbate inflammation including antibiotic resistance and adhesion mechanisms [32]. H. Parainfluanzae was particularly identified on biopsy as an active constituent in UC patients, correlating with disease severity and treatment efficacy [32]. Specifically, in CD treated with infliximab, as in the Eun et al. study, the abundance of Gammaproteobacteria was proportionally increased in both fecal and mucosal tissue samples [36]. Some animal models suggest the microbiome causes an adjuvant effect that primes autoreactive adaptive immune responses even in extraintestinal sites [33]. Whether microbial participation is a cause or consequence of UC, it is innately tied to the pathophysiology of IBD as microbial biomarkers are also predictive of treatment efficacy and may have a trajectory in furthering machine learning as a predictive tool, as described by Sakurai et al. $[28,32]$ Further work to understand microbiome profiles in both diseased and healthy states may elucidate our understanding of treatment non-responders, as intestinal-level metabolism impacts drug bioavailability [29].

Limitations

There are some limitations to this study as only work published in the English language in the past six years involving human subjects was evaluated. We may have missed studies published in other languages outside of this time frame, which may impact the data available to us. Both the genetic constitution and dietary habits of the human population have been shown to impact the development of IBD and gut microbial communities, therefore, we may have missed other valuable studies published in different languages which may assess this topic in different cultural populations. This study focused on the involvement of human subjects, however, exclusively animal models not included here may provide further insight into disease process and treatment.

\section{Conclusions}

In this systematic review, we aimed to compile information on the interactions of the gut microbiome with immunotherapy in the course of disease and treatment of IBD patients. After thoroughly reviewing the studies, we found that although there are characteristic taxonomic shifts between diseased and healthy patients, genetic variants are an important consideration in the predictive quality of disease and treatment decisions. Despite genetic and dietary factors, the characteristic shifts in microbial populations were similar among the studied Asian and Western groups. Furthermore, the bioavailability of some treatments may be impacted by existing intestinal microbes; while intestinal microbial populations may change directly or indirectly in the course of treatment.

The juxtaposition between the interaction of microbial populations and treatment in addition to the existence of genetic variants may provide insight into why some patients do not respond fully to therapy. We believe that further categorization of microbial changes between diseased and healthy states will improve our therapeutic potential and prevention of both gastrointestinal and multi-system diseases. Limitations in our current knowledge including the non-bacterial components of the gut microcosm and the complexity of both the metagenomic gut composition and genetic variants of IBD need further clarity to improve our clinical predictive tools. Our study is necessary for furthering the need for specific characterization of microbial changes in IBD processes and treatment. Future studies should focus on the comparison between Western and other cultural populations as well as further implementation of GWAS in clinical predictability.

\section{Additional Information}

\section{Disclosures}

Conflicts of interest: In compliance with the ICMJE uniform disclosure form, all authors declare the following: Payment/services info: All authors have declared that no financial support was received from any organization for the submitted work. Financial relationships: All authors have declared that they have no financial relationships at present or within the previous three years with any organizations that might have an interest in the submitted work. Other relationships: All authors have declared that there are no other relationships or activities that could appear to have influenced the submitted work.

\section{References}

1. Petito V, Graziani C, Lopetuso LR, et al.: Anti-tumor necrosis factor $\alpha$ therapy associates to type 17 helper $\mathrm{T}$ lymphocytes immunological shift and significant microbial changes in dextran sodium sulphate colitis. World J Gastroenterol. 2019, 25:1465-77. 10.3748/wjg.v25.i12.1465

2. Babickova J, Gardlik R: Pathological and therapeutic interactions between bacteriophages, microbes and the host in inflammatory bowel disease. World J Gastroenterol. 2015, 21:11321-30. 10.3748/wjg.v21.i40.11321

3. Hodzic Z, Schill EM, Bolock AM, Good M: IL-33 and the intestine: The good, the bad, and the inflammatory . Cytokine. 2017, 100:1-10. 10.1016/j.cyto.2017.06.017

4. He C, Wang H, Liao WD, Peng C, Shu X, Zhu X, Zhu ZH: Characteristics of mucosa-associated gut microbiota during treatment in Crohn's disease. World J Gastroenterol. 2019, 25:2204-16. 
10.3748/wig.v25.i18.2204

5. Bäumler AJ, Sperandio V: Interactions between the microbiota and pathogenic bacteria in the gut . Nature. 2016, 535:85-93. 10.1038/nature18849

6. Zhou M, He J, Shen Y, Zhang C, Wang J, Chen Y: New frontiers in genetics, gut microbiota, and immunity: a Rosetta Stone for the pathogenesis of inflammatory bowel disease. Biomed Res Int. 2017, 2017:8201672. $10.1155 / 2017 / 8201672$

7. Davenport ER, Cusanovich DA, Michelini K, Barreiro LB, Ober C, Gilad Y: Genome-wide association studies of the human gut microbiota. PLoS One. 2015, 10:e0140301. 10.1371/journal.pone.0140301

8. Franzosa EA, Sirota-Madi A, Avila-Pacheco J, et al.: Gut microbiome structure and metabolic activity in inflammatory bowel disease. Nat Microbiol. 2019, 4:293-305. 10.1038/s41564-018-0306-4

9. Vrakas S, Mountzouris KC, Michalopoulos G, Karamanolis G, Papatheodoridis G, Tzathas C, Gazouli M: Intestinal bacteria composition and translocation of bacteria in inflammatory bowel disease . PLoS One. 2017, 12:e0170034. 10.1371/journal.pone.0170034

10. Viennois E, Gewirtz AT, Chassaing B: Chronic inflammatory diseases: are we ready for microbiota-based dietary intervention?. Cell Mol Gastroenterol Hepatol. 2019, 8:61-71. 10.1016/j.jcmgh.2019.02.008

11. Wang W, Jovel J, Halloran B, et al.: Metagenomic analysis of microbiome in colon tissue from subjects with inflammatory bowel diseases reveals interplay of viruses and bacteria. Inflamm Bowel Dis. 2015, 21:141927. 10.1097/MIB.0000000000000344

12. Zeber-Lubecka N, Kulecka M, Ambrozkiewicz F, et al.: Limited prolonged effects of rifaximin treatment on irritable bowel syndrome-related differences in the fecal microbiome and metabolome. Gut Microbes. 2016, 7:397-413. 10.1080/19490976.2016.1215805

13. Jones-Hall YL, Nakatsu CH: The intersection of TNF, IBD and the microbiome . Gut Microbes. 2016, 7:58-62. 10.1080/19490976.2015.1121364

14. Kordy K, Romeo AC, Lee DJ, et al.: Combination antibiotics improves disease activity and alters microbial communities in children with ulcerative colitis. J Pediatr Gastroenterol Nutr. 2018, 67:e60-3. 10.1097/MPG.0000000000002034

15. Alipour M, Zaidi D, Valcheva R, et al.: Mucosal barrier depletion and loss of bacterial diversity are primary abnormalities in paediatric ulcerative colitis. J Crohns Colitis. 2016, 10:462-71. 10.1093/ecco-jcc/jjv223

16. Fernandes MA, Verstraete SG, Phan TG, et al.: Enteric virome and bacterial microbiota in children with ulcerative colitis and Crohn disease. J Pediatr Gastroenterol Nutr. 2019, 68:30-6. 10.1097/MPG.0000000000002140

17. Li XV, Leonardi I, Iliev ID: Gut mycobiota in immunity and inflammatory disease . Immunity. 2019, 50:136579. 10.1016/j.immuni.2019.05.023

18. James SC, Fraser K, Young W, McNabb WC, Roy NC: Gut microbial metabolites and biochemical pathways involved in irritable bowel syndrome: effects of diet and nutrition on the microbiome. J Nutr. 2020, 150:1012-21. 10.1093/jn/nxz302

19. Kim SK, Guevarra RB, Kim YT, et al.: Role of probiotics in human gut microbiome-associated diseases. J Microbiol Biotechnol. 2019, 29:1335-40. 10.4014/imb.1906.06064

20. Ho JT, Chan GC, Li JC: Systemic effects of gut microbiota and its relationship with disease and modulation . BMC Immunol. 2015, 16:21. 10.1186/s12865-015-0083-2

21. Sham HP, Bazett M, Bosiljcic M, et al.: Immune stimulation using a gut microbe-based immunotherapy reduces disease pathology and improves barrier function in ulcerative colitis. Front Immunol. 2018, 9:2211. 10.3389/fimmu.2018.02211

22. Schierová D, Březina J, Mrázek J, Fliegerová KO, Kvasnová S, Bajer L, Drastich P: Gut microbiome changes in patients with active left-sided ulcerative colitis after fecal microbiome transplantation and topical 5aminosalicylic acid therapy. Cells. 2020, 9:10.3390/cells9102283

23. Moulton CD, Pavlidis P, Norton C, Norton S, Pariante C, Hayee B, Powell N: Depressive symptoms in inflammatory bowel disease: an extraintestinal manifestation of inflammation?. Clin Exp Immunol. 2019, 197:308-18. 10.1111/cei.13276

24. Chen DL, Dai YC, Zheng L, Chen YL, Zhang YL, Tang ZP: Features of the gut microbiota in ulcerative colitis patients with depression: A pilot study. Medicine (Baltimore). 2021, 100:e24845. 10.1097/MD.0000000000024845

25. Wang SL, Shao BZ, Zhao SB, et al.: Intestinal autophagy links psychosocial stress with gut microbiota to promote inflammatory bowel disease. Cell Death Dis. 2019, 10:391. 10.1038/s41419-019-1634-X

26. Page MJ, McKenzie JE, Bossuyt PM, et al.: The PRISMA 2020 statement: an updated guideline for reporting systematic reviews. BMJ. 2021, 372:n71. 10.1136/bmj.n71

27. PubMed. (2021). Accessed: April 29, 2021: https://pubmed.ncbi.nlm.nih.gov/.

28. Sakurai T, Nishiyama H, Sakai K, et al.: Mucosal microbiota and gene expression are associated with longterm remission after discontinuation of adalimumab in ulcerative colitis. Sci Rep. 2020, 10:19186. 10.1038/s41598-020-76175-2

29. O'Reilly C, O'Sullivan Ó, Cotter PD, et al.: Encapsulated cyclosporine does not change the composition of the human microbiota when assessed ex vivo and in vivo. J Med Microbiol. 2020, 69:854-63. 10.1099/jmm.0.001130

30. Li E, Zhang Y, Tian X, et al.: Influence of Crohn's disease related polymorphisms in innate immune function on ileal microbiome. PLoS One. 2019, 14:e0213108. 10.1371/journal.pone.0213108

31. Carstens A, Dicksved J, Nelson R, et al.: The cut microbiota in collagenous colitis shares characteristics with inflammatory bowel disease-associated dysbiosis. Clin Transl Gastroenterol. 2019, 10:e00065. 10.14309/ctg.0000000000000065

32. Schirmer M, Denson L, Vlamakis H, et al.: Compositional and temporal changes in the gut microbiome of pediatric ulcerative colitis patients are linked to disease course. Cell Host Microbe. 2018, 24:600-610.e4. 10.1016/j.chom.2018.09.009

33. Grigg JB, Sonnenberg GF: Host-microbiota interactions shape local and systemic inflammatory diseases . J Immunol. 2017, 198:564-71. 10.4049/jimmunol.1601621

34. Guariso G, Gasparetto M: Treating children with inflammatory bowel disease: Current and new 


\section{Cureus}

perspectives. World J Gastroenterol. 2017, 23:5469-85. 10.3748/wig.v23.i30.5469

35. Agus A, Denizot J, Thévenot J, et al.: Western diet induces a shift in microbiota composition enhancing susceptibility to Adherent-Invasive E. coli infection and intestinal inflammation. Sci Rep. 2016, 6:19032. 10.1038/srep19032

36. Eun CS, Kwak MJ, Han DS, et al.: Does the intestinal microbial community of Korean Crohn's disease patients differ from that of western patients?. BMC Gastroenterol. 2016, 16:28. 10.1186/s12876-016-0437-0

37. Lewis JD, Chen EZ, Baldassano RN, et al.: Inflammation, antibiotics, and diet as environmental stressors of the gut microbiome in pediatric Crohn's disease. Cell Host Microbe. 2015, 18:489-500.

10.1016/j.chom.2015.09.008 